\title{
Evaluation of disease severity in bronchiectasis using impulse oscillometry
}

Yuji Yamamoto (D, Keisuke Miki, Kazuyuki Tsujino, Tomoki Kuge, Takanori Matsuki, Kiyoharu Fukushima, Yohei Oshitani, Hiroyuki Kagawa, Kenji Yoshimura, Mari Miki and Hiroshi Kida

Affiliation: Dept of Respiratory Medicine, National Hospital Organization Osaka Toneyama Medical Center, Toyonaka, Japan.

Correspondence: Yuji Yamamoto, Dept of Respiratory Medicine, National Hospital Organization Osaka Toneyama Medical Center, 5-1-1 Toneyama, Toyonaka, Osaka, 560-8552, Japan.

E-mail: cyyamamoto1110agmail.com

ABSTRACT Although the diagnostic value of impulse oscillometry (IOS) in bronchiectasis for the differential diagnosis of healthy subjects has been researched, the usefulness of each IOS parameter for predicting disease severity in bronchiectasis has not been thoroughly investigated. In addition, the usefulness of IOS in patients with nontuberculous mycobacteria (NTM) infection has not been reported. This study aimed to determine the predictive significance of respiratory impedance and detect the other most significant IOS parameters for predicting disease severity in bronchiectasis patients and to validate the usefulness of IOS in patients with NTM infection.

A total of 206 patients with bronchiectasis who attended clinics at the National Hospital Organization Osaka Toneyama Medical Center were included. Chest high-resolution computed tomography, spirometry and IOS were performed. Hospital admissions, mortality and disease severity indices for bronchiectasis (Bronchiectasis Severity Index (BSI), FACED, and E-FACED scores) were calculated to assess disease severity. The patients were divided into subgroups with and without NTM infection, and subgroup analyses were performed.

Respiratory reactance, especially resonant frequency $\left(f_{\text {res }}\right)$, correlated with both BSI and FACED score better than respiratory resistance. Inspiratory but not expiratory impedance was strongly correlated with BSI, FACED and E-FACED scores. Inspiratory $f_{\text {res }}$ was the most useful predictor, increasing as the disease became more severe. The usefulness of IOS was almost equivalent in patients both with and without NTM infection.

Inspiratory reactance measured by IOS is useful for estimating disease severity in bronchiectasis. Inspiratory $f_{\text {res }}$ best predicts disease severity in bronchiectasis patients both with and without NTM infection.

@ERSpublications

Inspiratory reactance measured by impulse oscillometry may be useful for estimating disease severity of bronchiectasis. Inspiratory $f_{\text {res }}$ can be used to evaluate severity in the disease both with and without NTM infection. https://bit.ly/3fvG0Rn

Cite this article as: Yamamoto $\mathrm{Y}$, Miki $\mathrm{K}$, Tsujino $\mathrm{K}$, et al. Evaluation of disease severity in bronchiectasis using impulse oscillometry. ERJ Open Res 2020; 6: 00053-2020 [https://doi.org/ 10.1183/23120541.00053-2020].

This article has supplementary material available from openres.ersjournals.com

Received: 3 Feb 2020 | Accepted after revision: 7 July 2020

Copyright $\odot$ ERS 2020. This article is open access and distributed under the terms of the Creative Commons Attribution Non-Commercial Licence 4.0. 


\section{Introduction}

Bronchiectasis is a chronic respiratory disease. Its symptoms include recurrent cough, sputum and respiratory infections [1]. The host-mediated inflammatory response to foreign material and bacteria induces tissue damage and subsequent bronchiectasis, which is associated with abnormal mucus clearance and bacterial colonisation [2, 3]. In particular, Pseudomonas aeruginosa colonisation (Pseudomonas colonisation) is one of the independent predictors of poor prognosis [4-7]. Although there was no accepted definition of the disease severity of bronchiectasis, Bronchiectasis Severity Index (BSI), FACED and E-FACED scores have been developed $[6,8,9]$. These are novel scores with great accuracy that combine spirometry and the Medical Research Council (MRC) dyspnoea scale, which can evaluate various clinical outcomes, including hospital admissions and mortality $[6,8,10]$. However, the scores are difficult to obtain from patients at the first visit and without regular visits because they require several sputum cultures to confirm bacterial colonisation and the BSI and E-FACED score further require histories of exacerbations and hospital admissions. Therefore, pulmonary function tests (PFTs), including spirometry and impulse oscillometry (IOS), could remain important for evaluating disease severity in bronchiectasis effortlessly.

IOS, which involves measurements of within-breath changes in respiratory impedance, measures respiratory resistance $\left(R_{\mathrm{rs}}\right)$ and respiratory reactance $\left(X_{\mathrm{rs}}\right) . R_{\mathrm{rs}}$ represents the sum of the airway resistance and viscous resistance of lung and thoracic tissue [11], while $X_{\mathrm{rs}}$ reflects the dynamic elastance and inertia of the respiratory system [12]. Measurement of respiratory impedance by IOS is less time-consuming and technically easier to perform than spirometry because it is measured at rest under conditions of minimal respiratory effort [13]. In addition, IOS can show complementary information of the lungs and might be useful in the assessment of respiratory mechanics [14].

Regarding bronchiectasis, the majority of reports for IOS are in paediatric cystic fibrosis (CF) populations, with reports of the disease in adults being rare [13, 15]. GuAn et al. [16] first reported the usefulness of IOS as a tool for differentiating bronchiectasis patients from healthy subjects in adults. The study revealed that an increased number of abnormal IOS parameters indicates poor clinical conditions [16]. They also demonstrated that IOS had a high diagnostic value for mild-to-moderate bronchiectasis [17]. In particular, IOS parameters that indicate small airways were reportedly useful for the diagnosis [17]. Despite these preceding studies, the usefulness of IOS for evaluating the disease severity of bronchiectasis in adults has never been thoroughly investigated. In addition, the usefulness of IOS for patients with nontuberculous mycobacteria (NTM) infection has never been reported. The information obtained from IOS might be a useful tool for predicting the clinical outcomes of bronchiectasis adult patients and assessing the respiratory physiology of bronchiectasis.

The objectives of the present study were as follows: 1) to investigate the usefulness of IOS as a tool for predicting two important clinical outcomes in patients with bronchiectasis: hospital admissions and mortality; 2) to identify any IOS parameters significant for predicting hospital admissions, mortality, and the severity indices associated with bronchiectasis; and 3) to assess the potential relationships between NTM infection and IOS parameters, hospital admissions and mortality in bronchiectasis patients.

\section{Methods}

Subjects

A total of 405 Japanese patients with bronchiectasis who attended clinics at the National Hospital Organization (NHO) Osaka Toneyama Medical Center between February 2013 and October 2019 were screened in this study. The patients were diagnosed with bronchiectasis based on their clinical symptoms (cough, purulent sputum and haemoptysis) and chest high-resolution computed tomography (HRCT) findings. Patients who did not undergo spirometry or IOS within 3 months from the time of diagnosis were excluded. Moreover, patients were excluded if they had CF, malignant tumours, severe heart diseases, severe cerebral diseases or primary diagnoses of pulmonary fibrosis/sarcoidosis with secondary traction bronchiectasis. The qualified 206 patients underwent the examinations and evaluations described in the following sections.

\section{Study design}

Respiratory impedance was measured in all patients using IOS, and spirometry was performed on the same day. Short-acting $\beta_{2}$-agonists were not used for at least $12 \mathrm{~h}$ before tests in all patients. Long-acting $\beta_{2}$-agonists (LABAs) and long-acting antimuscarinic agents (LAMAs) were not withdrawn before the measurement of PFTs. Chest HRCT scans were conducted with 1-mm section thickness and within 12 months from the IOS and spirometric measurements. Bacterial colonisation was defined as the presence of at least two sputum of the same pathogen at least 3 months apart in a 1-year period [18]. The patients were divided into a subgroup with NTM infection (NTM subgroup) and without NTM infection 
(non-NTM subgroup) based on the presence of symptoms, radiological abnormalities, and microbiological cultures that excluded other potential aetiologies [19]. Correlations between IOS parameters, spirometric parameters and the disease severity indices (BSI, FACED and E-FACED scores) were analysed. The IOS parameters were then compared with the disease severity indices for their usefulness in predicting disease severity. This comparison was made regarding the total patients, the NTM subgroup and the non-NTM subgroup. The Institutional Review Board of the NHO Osaka Toneyama Medical Center approved the study protocols and chose an opt-out system for obtaining patients' informed consent (approval number: TNH-2019042).

\section{Measurement of respiratory impedance using IOS}

Respiratory impedance was measured at rest with broadband IOS using a commercially available device (Mostgraph-01, Chest M.I. Co., Ltd., Tokyo, Japan). The methods were performed according to the standard recommendations [11]. Partitioned (inspiratory and expiratory) and average IOS parameters were measured. As indicators of the frequency dependence of $R_{\mathrm{rs}}, R_{\mathrm{rs}}$ at 5 and $20 \mathrm{~Hz}\left(R_{5}\right.$ and $R_{20}$, respectively) and the difference between these $\left(R_{5}-R_{20}\right)$ were used. In addition, $X_{\mathrm{rs}}$ at $5 \mathrm{~Hz}\left(X_{5}\right)$, resonant frequency $\left(f_{\text {res }}\right)$ and low-frequency reactance area (ALX) were used as indicators of respiratory reactance. $f_{\text {res }}$ indicates the point at which $X_{\mathrm{rs}}$ crosses zero and elasticity and inertia balance each other, and ALX is defined as the integral of $X_{5}$ to $f_{\text {res }}[14]$.

\section{Spirometry}

The patients underwent spirometry using the CHESTAC 8800 spirometer (Chest MI, Inc., Tokyo, Japan) according to the recommendations of the American Thoracic Society [20]. The predicted vital capacity $(\mathrm{VC})$ and forced expiration volume in $1 \mathrm{~s}\left(\mathrm{FEV}_{1}\right)$ were calculated according to the formula for Japanese subjects developed by the Japanese Respiratory Society [21].

\section{The BSI, FACED and E-FACED scores}

The BSI, FACED and E-FACED scores were calculated for all patients in the present study. All indices included age, $\mathrm{MRC} /$ modified MRC dyspnoea scale, $\mathrm{FEV}_{1} \%$ predicted, number of pulmonary lobes with bronchiectasis and Pseudomonas colonisation [6, 8, 9]. The BSI incorporated body mass index (BMI), exacerbations, hospital admissions and colonisation with other organisms, in addition to the variables described above, and was calculated by summing the scores of each. The final BSI score ranged from 0 to 26, with a score between 0 and 4 indicating a mild case of the disease, between 5 and 8 indicating a moderate case, and 9 or more indicating a severe case [6]. The FACED score incorporated the five abovementioned variables and was calculated by summing the scores of each. The final FACED score ranged from 0 to 7 , with a score between 0 and 2 indicating a mild case of the disease, between 3 and 4 indicating a moderate case, and between 5 and 7 indicating a severe case [8]. The E-FACED score was calculated by adding the score of hospital admissions to the FACED score. The final E-FACED score ranged from 0 to 9 , with a score between 0 and 3 indicating a mild case of the disease, between 4 and 6 indicating a moderate case, and between 7 and 9 indicating a severe case $[8,9]$.

\section{Assessment of disease severity of bronchiectasis}

The disease severity of bronchiectasis was assessed according to hospital admissions and mortality. Exacerbations were defined as the patients' status that requires antibiotic therapy, (i.e. the presence of increased sputum purulence, increased sputum volume or change in viscosity) and increased pulmonary symptoms [22]. Unscheduled hospital admissions due to exacerbations of bronchiectasis were recorded [22]. Exacerbations, hospital admissions due to exacerbations and survival status were measured for all patients within 4 years from their first visit. In cases of mortality, cause of death was determined and assigned as bronchiectasis related or unrelated [6]. All the data were collected by individual case review.

\section{Statistical analysis}

The Mann-Whitney $U$-test, t-test and Chi-squared test were used for comparison of IOS parameters among the two subgroups and necessary sample size was calculated to present the statistical power. The Kruskal-Wallis test was used for comparison of IOS parameters among patients with mild-to-severe bronchiectasis stratified by the BSI, FACED and E-FACED scores. Spearman's rank correlation coefficient and univariate analyses were used for bivariate correlation analysis between IOS parameters, spirometric parameters, the BSI, FACED and E-FACED scores. Multiple stepwise linear regression analysis was performed to detect IOS parameters that were significant determinants of the BSI, FACED and E-FACED scores. After detecting the most significant IOS parameters, the receiver operating characteristic (ROC) curves of these IOS parameters were compared to $\mathrm{FEV}_{1}$, the BSI, FACED and E-FACED scores for predicting hospital admissions and mortality using the DeLong test [23]. Univariate and multivariate analyses and the Mann-Whitney $U$-test were used for subgroup and interaction analyses. For all analyses, 


\section{TABLE 1 Patients' baseline characteristics ( $n=206$ )}

Parameter

Age years

Male/female $n$

Height cm

Weight kg

BMI $\mathrm{kg} \cdot \mathrm{m}^{-2}$

Never-smokers

Medications

LAMA

LABA

ICS

Theophylline

Macrolides

Mucolytics

Underlying cause

MAC

Idiopathic/post-infective

Previous TB

Other NTM

Rheumatoid arthritis

ABPA

Others

\section{Bronchiectasis patients}

$$
\begin{gathered}
70.55 \pm 11.09 \\
64 / 142 \\
157.3 \pm 8.2 \\
49.6 \pm 10.0 \\
19.95 \pm 3.18 \\
142(68.93) \\
\\
33(16.02) \\
54(26.21) \\
50(24.27) \\
15(7.28) \\
140(67.96) \\
129(62.62) \\
\\
86(41.75) \\
74(35.92) \\
17(8.25) \\
12(5.83) \\
11(5.34) \\
3(1.46) \\
3(1.46)
\end{gathered}
$$

\begin{tabular}{|c|c|c|}
\hline \multirow{2}{*}{$\begin{array}{l}\text { Parameter } \\
\text { Spirometry }\end{array}$} & \multicolumn{2}{|c|}{ Bronchiectasis patients } \\
\hline & & \\
\hline $\mathrm{FEV} V_{1} \mathrm{~L}$ & $1.59 \pm 0.68$ & 0.53 to 3.75 \\
\hline $\mathrm{FEV}_{1} \%$ predicted & $75.2 \pm 25.4$ & 18.2 to 131.1 \\
\hline $\mathrm{FEV}_{1} / \mathrm{FVC} \%$ & $70.9 \pm 15.0$ & 27.6 to 100.0 \\
\hline VC L & $2.26 \pm 0.85$ & 0.64 to 4.65 \\
\hline VC \% & $80.1 \pm 22.2$ & 22.3 to 121.7 \\
\hline Three or more exacerbations per year & $40(20.93)$ & \\
\hline Hospital admission within 4 years & $91(44.17)$ & \\
\hline Mortality within 4 years & $20(9.71)$ & \\
\hline MRC dyspnoea score & $2.38 \pm 1.41$ & \\
\hline Pseudomonas colonisation & $54(26.21)$ & \\
\hline Colonisation with other organisms & $143(69.42)$ & \\
\hline Pulmonary lobes with bronchiectasis or cystic bronchiectasis $n$ & $3.32 \pm 1.33$ & \\
\hline Bronchiectasis Severity Index & $10.18 \pm 5.42$ & \\
\hline Mild $(0-4)$ & $33(16.02)$ & \\
\hline Moderate (5-8) & $53(25.73)$ & \\
\hline Severe ( $>9$ or more) & $120(58.25)$ & \\
\hline FACED score & $2.76 \pm 1.78$ & \\
\hline Mild (0-2) & $90(43.69)$ & \\
\hline Moderate (3-4) & 84 (40.78) & \\
\hline Severe (5-7) & $32(15.53)$ & \\
\hline E-FACED score & $3.15 \pm 2.17$ & \\
\hline Mild & $126(61.17)$ & \\
\hline Moderate & $61(29.61)$ & \\
\hline Severe & $19(9.22)$ & \\
\hline
\end{tabular}

Data are presented as mean \pm SD along with the minimum and maximum values (right hand column) or $\mathrm{n}(\%)$, unless otherwise stated. BMI: body mass index; LABA: long-acting $\beta_{2}$-agonist; LAMA: long-acting muscarinic antagonist; ICS: inhaled corticosteroid; MAC: Mycobacterium avium complex; TB: tuberculosis; NTM: nontuberculous mycobacteria; ABPA: allergic bronchopulmonary aspergillosis.

\section{TABLE 2 Clinical characteristics of bronchiectasis patients ( $n=206$ )}

Data are presented as mean $\pm \mathrm{SD}$ along with the minimum and maximum values (right hand column) or $\mathrm{n}$ $(\%)$, unless otherwise stated. $F_{1}$ : forced expiratory volume in $1 \mathrm{~s}$; FVC: forced vital capacity; VC: vital capacity; MRC: Medical Research Council; Pseudomonas colonisation: Pseudomonas aeruginosa colonisation. 


\begin{tabular}{|c|c|c|c|}
\hline Parameter & $\begin{array}{l}\text { Non-NTM subgroup } \\
\qquad(n=108)\end{array}$ & $\begin{array}{l}\text { NTM subgroup } \\
\qquad(n=98)\end{array}$ & p-value \\
\hline Age years & $70.74 \pm 12.12$ & $70.34 \pm 9.88$ & 0.7947 \\
\hline Male/female $n$ & $43 / 65$ & $21 / 77$ & 0.0043 \\
\hline BMI $\mathrm{kg} \cdot \mathrm{m}^{-2}$ & $20.25 \pm 3.42$ & $19.62 \pm 2.87$ & 0.1542 \\
\hline VC $\%$ & $75.53 \pm 23.49$ & $85.21 \pm 19.55$ & 0.0016 \\
\hline $\mathrm{FEV}_{1} \%$ predicted & $70.92 \pm 27.34$ & $79.95 \pm 22.27$ & 0.0104 \\
\hline Three or more exacerbations per year & $21(19.44)$ & 19 (19.39) & 0.9919 \\
\hline Hospital admission within 4 years & 53 (49.07) & 38 (38.78) & 0.1385 \\
\hline Mortality within 4 years & $14(12.96)$ & $6(6.12)$ & 0.0986 \\
\hline MRC dyspnoea score & $2.65 \pm 1.48$ & $2.08 \pm 1.27$ & 0.0038 \\
\hline Pseudomonas colonisation & $44(40.74)$ & $10(10.20)$ & $<0.0001$ \\
\hline Colonisation with other organisms & $47(43.52)$ & $98(100.00)$ & $<0.0001$ \\
\hline $\begin{array}{l}\text { Pulmonary lobes with bronchiectasis or cystic } \\
\text { bronchiectasis }\end{array}$ & $3.39 \pm 1.37$ & $3.24 \pm 1.30$ & 0.4405 \\
\hline Bronchiectasis Severity Index & $11.05 \pm 5.68$ & $9.23 \pm 4.99$ & 0.0163 \\
\hline Mild (0-4) & 15 (13.89) & $18(18.37)$ & 0.1370 \\
\hline Moderate (5-8) & $23(21.30)$ & $30(30.61)$ & \\
\hline Severe (>9 or more) & 70 (64.81) & 50 (51.02) & \\
\hline FACED score & $3.17 \pm 1.85$ & $2.31 \pm 1.59$ & 0.0005 \\
\hline Mild (0-2) & $38(35.19)$ & $52(53.06)$ & 0.0049 \\
\hline Moderate (3-4) & $46(42.59)$ & 38 (38.78) & \\
\hline Severe (5-7) & $24(22.22)$ & $8(8.16)$ & \\
\hline E-FACED score & $3.56 \pm 2.25$ & $2.69 \pm 2.01$ & 0.0043 \\
\hline Mild & $57(52.78)$ & $69(70.41)$ & 0.0228 \\
\hline Moderate & $37(34.26)$ & $24(24.49)$ & \\
\hline Severe & $14(12.96)$ & $5(5.10)$ & \\
\hline
\end{tabular}

Data are presented as mean \pm SD or $\mathrm{n}(\%)$, unless otherwise stated. BMI: body mass index; VC: vital capacity; FEV 1 : forced expiratory volume in $1 \mathrm{~s} ;$ MRC: Medical Research Council; Pseudomonas colonisation: Pseudomonas aeruginosa colonisation.

p-values $<0.05$ were considered statistically significant. All statistical analyses were performed using EZR version 1.38 (based on $R$ version 3.5.2) and R commander version 2.5-1, Saitama, Japan; [24].

\section{Results}

\section{Baseline characteristics}

Among the 206 patients included in the present study, 98 patients were in the NTM subgroup, while 108 were in the non-NTM subgroup. Many of the patients had been treated with LAMAs, LABAs, inhaled corticosteroids (ICSs), theophylline, macrolides and/or mucolytics (table 1). Overall, 20 mortalities (9.71\%) and 44 hospital admissions (44.17\%) were recorded within 4 years of the first patients' initial visits. The use ratio of macrolides was high in this study compared with previous studies because the present study included patients with NTM colonisation or infection $[6,8,16]$. The average VC and $\mathrm{FEV}_{1} /$ forced vital capacity (FVC) were lower than VC (male, $3.37 \mathrm{~L}$; and female, $2.75 \mathrm{~L}$ ) and $\mathrm{FEV}_{1} / \mathrm{FVC}$ (male, $77.7 \%$; and female, 77.6\%) of healthy Japanese subjects (table 2, [21]). The baseline inspiratory IOS parameters indicated overall lower SD compared with expiratory parameters (supplementary table 1).

In the NTM subgroup, the ratio of females and $\mathrm{FEV}_{1} \%$ predicted were higher than in the non-NTM subgroup. The MRC dyspnoea scale, Pseudomonas colonisation, BSI, FACED and E-FACED scores were all lower than in the non-NTM subgroup (table 3). The frequency distributions of disease severity stratified by the BSI were not significantly different, but the mild disease ratio stratified by the FACED and E-FACED scores was higher in NTM subgroup (table 3). The difference was probably due to the scoring methods of age and Pseudomonas colonisation because the BSI weights these scores heavier than the FACED and E-FACED scores [6, 8,9]. There was no significant difference between the NTM and non-NTM subgroups for age, BMI, exacerbations, pulmonary lobes with bronchiectasis or cystic bronchiectasis, hospital admissions (38.78\% in the NTM subgroup and $49.07 \%$ in the non-NTM subgroup; $\mathrm{p}=0.1385)$ or mortality $(6.12 \%$ in the NTM subgroup and $12.96 \%$ in the non-NTM subgroup; $\mathrm{p}=0.0986$; table 3). Given that the necessary sample sizes required to present the statistical power were 768 for 

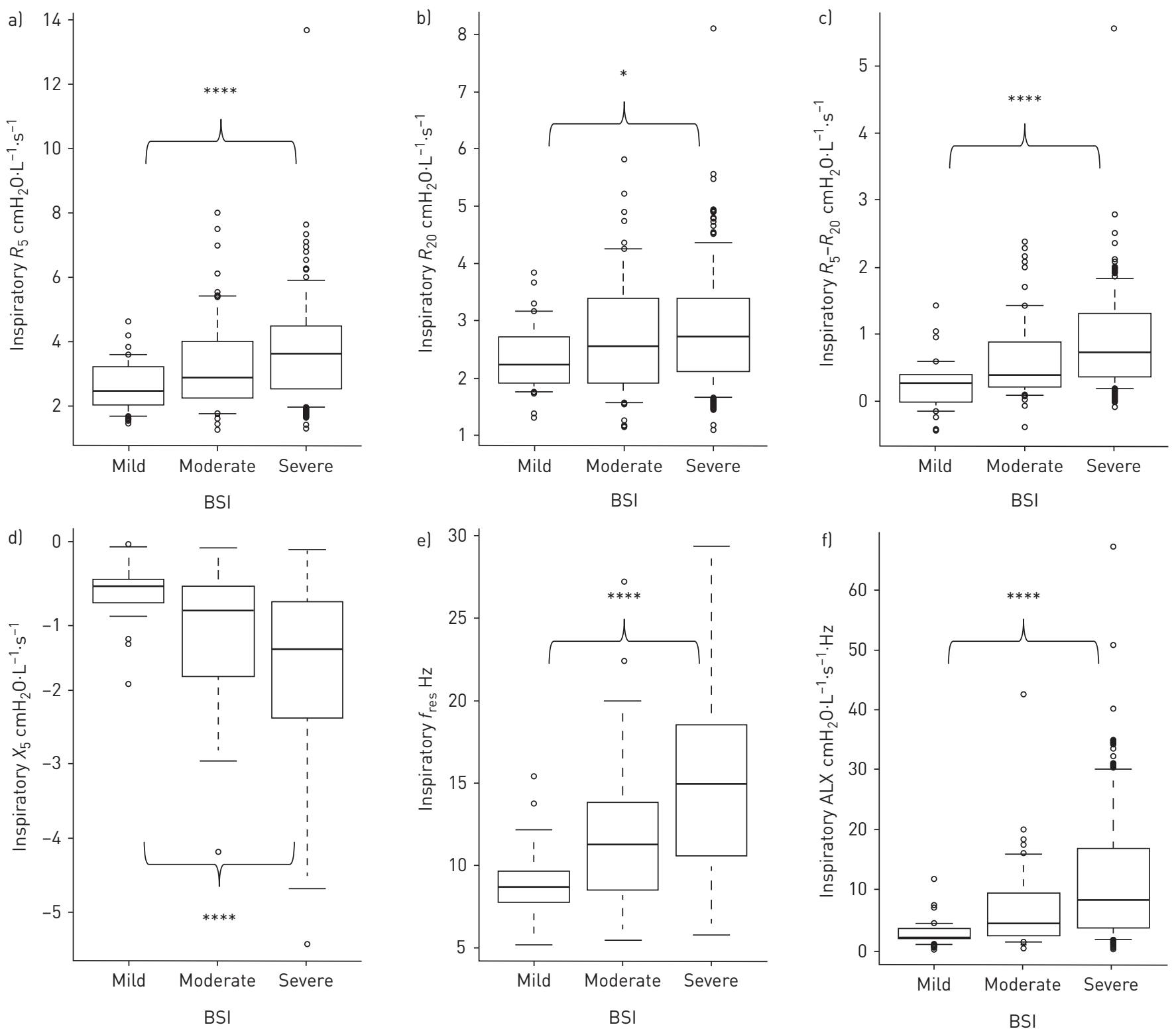

FIGURE 1 Results of inspiratory impedance at rest stratified by the Bronchiectasis Severity Index (BSI) (n=206). a) inspiratory respiratory system resistance at $\left.5 \mathrm{~Hz}\left(R_{5}\right), \mathrm{b}\right)$ inspiratory respiratory system resistance at $\left.20 \mathrm{~Hz}\left(R_{20}\right), \mathrm{c}\right)$ inspiratory $R_{5}-R_{20}$, d) inspiratory respiratory system reactance at $\left.5 \mathrm{~Hz}\left(X_{5}\right), e\right)$ inspiratory resonant frequency $\left(f_{\text {res }}\right)$, and $\left.f\right)$ inspiratory low-frequency reactance area (ALX). ${ }^{*}: p<0.05 ;{ }^{* * * *}: p<0.0001$, as measured by the Kruskal-Wallis test.

hospital admissions and 636 for mortality, the insignificant difference of hospital admissions and mortality between the two subgroups might have been attributed to the small number of subjects.

\section{Correlations of IOS parameters with spirometric parameters}

$\mathrm{VC}, \mathrm{FVC}$ and even $\mathrm{FEV}_{1}$ correlated the strongest with respiratory reactance (supplementary table 2). In addition, respiratory reactance correlated with $\mathrm{FEV}_{1} / \mathrm{FVC}$ stronger than respiratory resistance. This showed that respiratory reactance reflected restrictive ventilation deficiency and airflow obstruction in bronchiectasis.

\section{Identification of IOS parameters relevant to the BSI, FACED and E-FACED scores}

Respiratory reactance had a stronger correlation than respiratory resistance with the BSI, FACED and E-FACED scores (supplementary tables 3-5). Among resistance parameters, $R_{20}$ had the weakest correlation and expiratory $R_{20}$ showed no apparent correlation. $R_{5}-R_{20}$ had the strongest correlation of all the resistance parameters; however, even this correlation was weaker than respiratory reactance. Therefore, respiratory reactance rather than resistance correlated with the disease severity indices in bronchiectasis patients. 

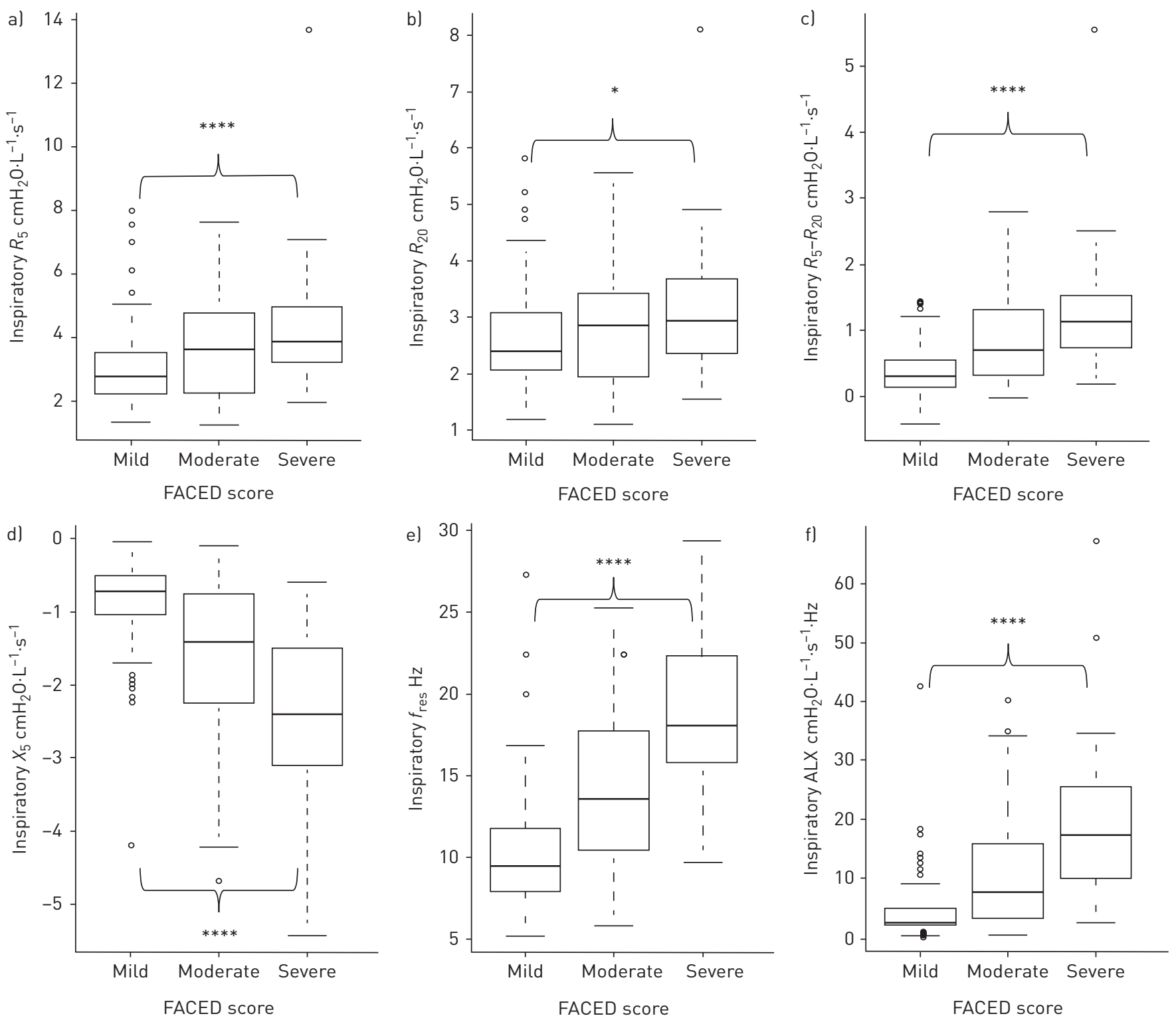

FIGURE 2 Results of inspiratory impedance at rest stratified by the FACED score $(\mathrm{n}=206)$. a) inspiratory respiratory system resistance at $5 \mathrm{~Hz}\left(R_{5}\right)$, b) inspiratory respiratory system resistance at $\left.20 \mathrm{~Hz}\left(R_{20}\right), \mathrm{c}\right)$ inspiratory $R_{5}-R_{20}$, d) inspiratory respiratory system reactance at $5 \mathrm{~Hz}\left(X_{5}\right)$, e) inspiratory resonant frequency $\left(f_{\text {res }}\right)$, and $\left.f\right)$ inspiratory low-frequency reactance area (ALX). *: $p<0.05$; $* * * *: p<0.0001$, as measured by the Kruskal-Wallis test.

Regarding reactance parameters, $f_{\text {res }}$ had the best correlation with all the disease severity indices (supplementary tables 3-5). Moreover, inspiratory but not expiratory impedance was correlated with both scores. Inspiratory IOS parameters stratified by the BSI and FACED and E-FACED scores are shown in figures 1 and 2, and supplementary figure 1. Multiple stepwise linear regression analyses also identified inspiratory $f_{\text {res }}$ as the most significant determinant of the disease severity indices (supplementary table 6). Overall, inspiratory $f_{\text {res }}$ was the most significant determinant of the disease severity indices.

\section{Detection of IOS parameters correlated with hospital admissions and mortality}

The ROC curves for predicting hospital admissions and mortality in bronchiectasis patients indicated that the area under curve (AUC) of respiratory reactance was higher than that of respiratory resistance (supplementary tables 7 and 8). Of note, the AUC of inspiratory $f_{\text {res }}$ was the highest of all the reactance parameters ( $\mathrm{AUC}=0.672$ for hospital admissions and $\mathrm{AUC}=0.781$ for mortality). The cut-off values of inspiratory $f_{\text {res }}$ for predicting hospital admissions and mortality were 13.120 and 14.180 , respectively $(\mathrm{p}<0.0001)$. Inspiratory rather than expiratory IOS parameters indicated higher AUC for predicting both hospital admissions and mortality, a trend similar to that shown in the analyses of the relationship 


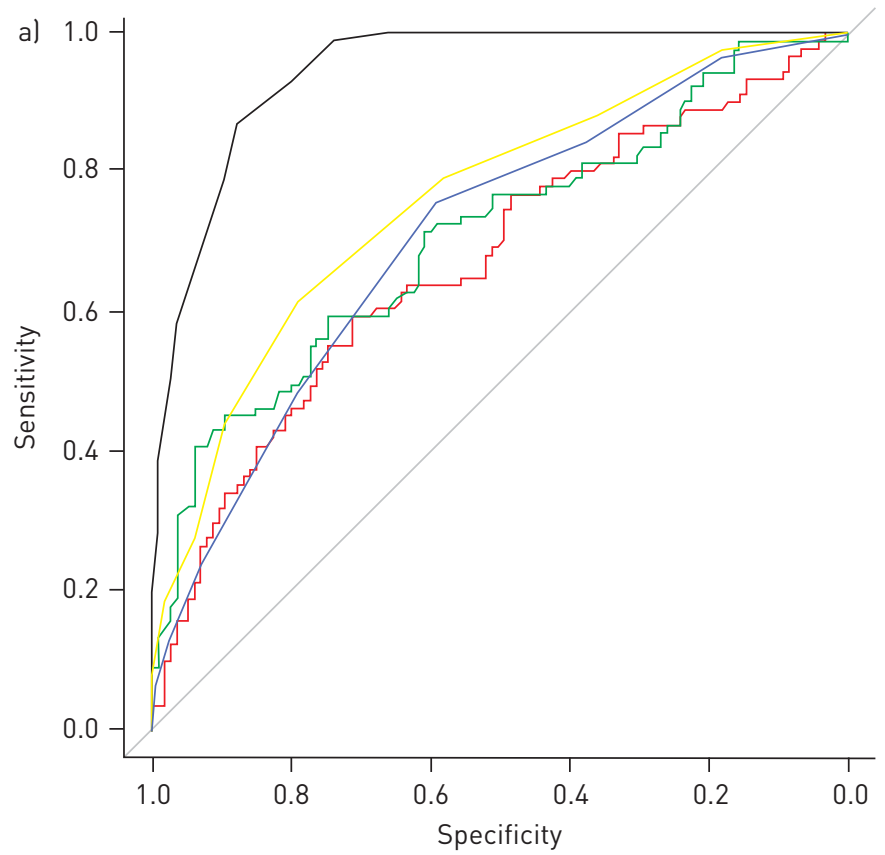

$\left.\begin{array}{l}\text { - Inspiratory } f_{\text {res }} \\ \text { AUC } 0.672(95 \% \mathrm{Cl} 0.597-0.747) \\ \left.\text { - } \begin{array}{l}\text { FEV } \\ \text { AUC } 0.710(95 \% \mathrm{Cl} 0.638-0.712) \\ \text { - FACED score } \\ \text { AUC } 0.712(95 \% \mathrm{Cl} 0.642-0.781)\end{array}\right] \text { NS } \\ \text { E-FACED score } \\ \text { AUC } 0.761(95 \% \mathrm{Cl} 0.696-0.826) \\ \text { - BSI } \\ \text { AUC } 0.944(95 \% \mathrm{Cl} 0.916-0.972)\end{array}\right\}_{* * * *}$

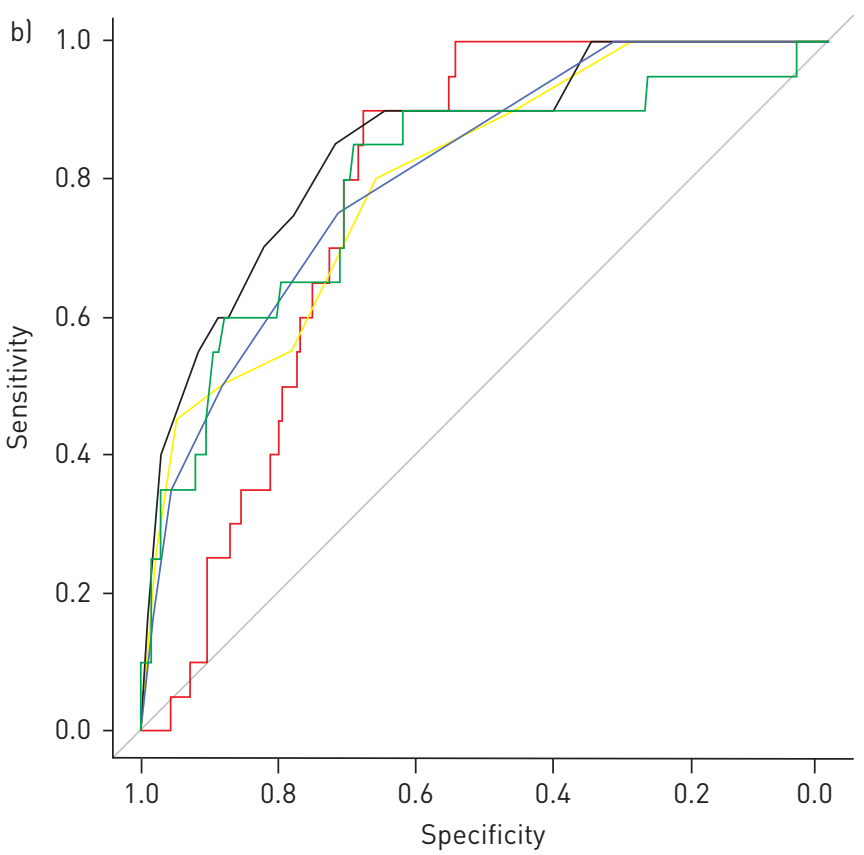

$\left.\begin{array}{l}\text { - Inspiratory } f_{\text {res }} \\ \text { AUC } 0.781(95 \% \mathrm{Cl} 0.711-0.850) \\ \text { - FEV } 1 \\ \text { AUC } 0.796(95 \% \mathrm{Cl} 0.681-0.912) \\ \text { - } \text { FACED score } \\ \text { AUC } 0.808(95 \% \mathrm{Cl} 0.711-0.900) \\ \text { - E-FACED score } \\ \text { AUC } 0.798(95 \% \mathrm{Cl} 0.702-0.895) \\ \text { - BSI } \\ \text { AUC } 0.849(95 \% \mathrm{Cl} 0.761-0.937)\end{array}\right\}$ NS

FIGURE 3 Receiver operating characteristic (ROC) curves for predicting hospital admissions and mortality ( $=206$ ). a) ROC curves for predicting hospital admissions within 4 years. b) ROC curves for predicting mortality within 4 years. AUC: area under curve; BSI: Bronchiectasis Severity Index; $\mathrm{FEV}_{1}$ : forced expiratory volume in $1 \mathrm{~s} ; f_{\text {res }}$ : resonant frequency; Ns: nonsignificant. $* * * *: p<0.0001$ as measured by the DeLong test.

between respiratory impedance and the disease severity indices. In addition, no treatment has significantly affected the correlation of inspiratory $f_{\text {res }}$ with hospital admissions and mortality (supplementary table 9). Given these analyses, inspiratory $f_{\text {res }}$ was identified as the most significant determinant of disease severity in bronchiectasis.

Comparison of the predictive usefulness of inspiratory $f_{\text {res, }} B S I$, FACED and E-FACED scores No apparent difference was observed between inspiratory $f_{\text {res }}$ (AUC $=0.672$ for hospital admissions and AUC $=0.781$ for mortality) and FACED score (AUC=0.712 for hospital admissions and AUC $=0.808$ for mortality, figure 3 ). Thus, the predictive usefulness of inspiratory $f_{\text {res }}$ was almost equivalent to FACED score. Although the AUC for hospital admissions was greater for BSI (AUC=0.944; $<<0.0001$ ), the AUC of inspiratory $f_{\text {res }}$ and BSI for mortality $(\mathrm{AUC}=0.849)$ were not significantly different $(\mathrm{p}=0.1368)$.

\section{Validation of inspiratory $\mathrm{f}_{\text {res }}$ for predicting disease severity in the NTM subgroup}

Between the subgroups, reactance but not resistance parameters were significantly different (figure 4 ). In particular, in the NTM subgroup, inspiratory $f_{\text {res }}$ and ALX were lower and $X_{5}$ was less negative (all p-values <0.05), indicating the better reactance of this subgroup. The AUC for predicting hospital admissions and mortality were almost equivalent between the NTM and non-NTM subgroups (table 4). The results demonstrated that inspiratory $f_{\text {res }}$ was useful for predicting disease severity in bronchiectasis patients with NTM infection.

Although the predictive usefulness was almost equivalent, the cut-off values of inspiratory $f_{\text {res }}$ for predicting hospital admissions and mortality were lower in the NTM subgroup (10.500 for hospital 

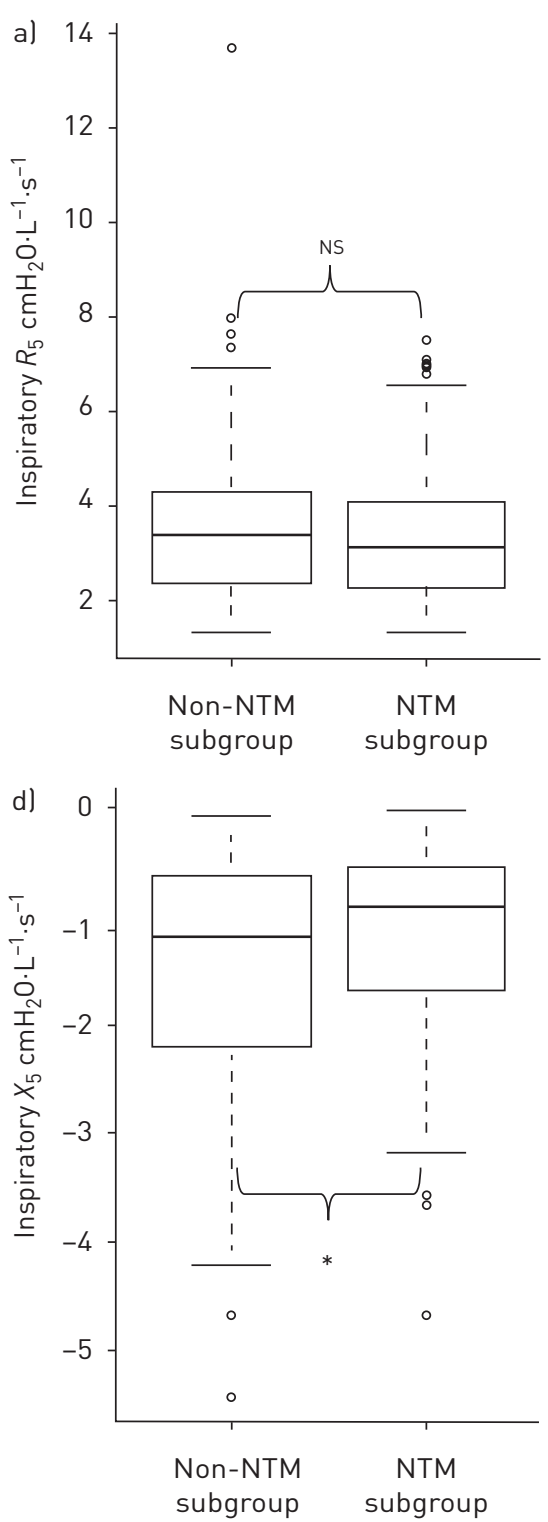
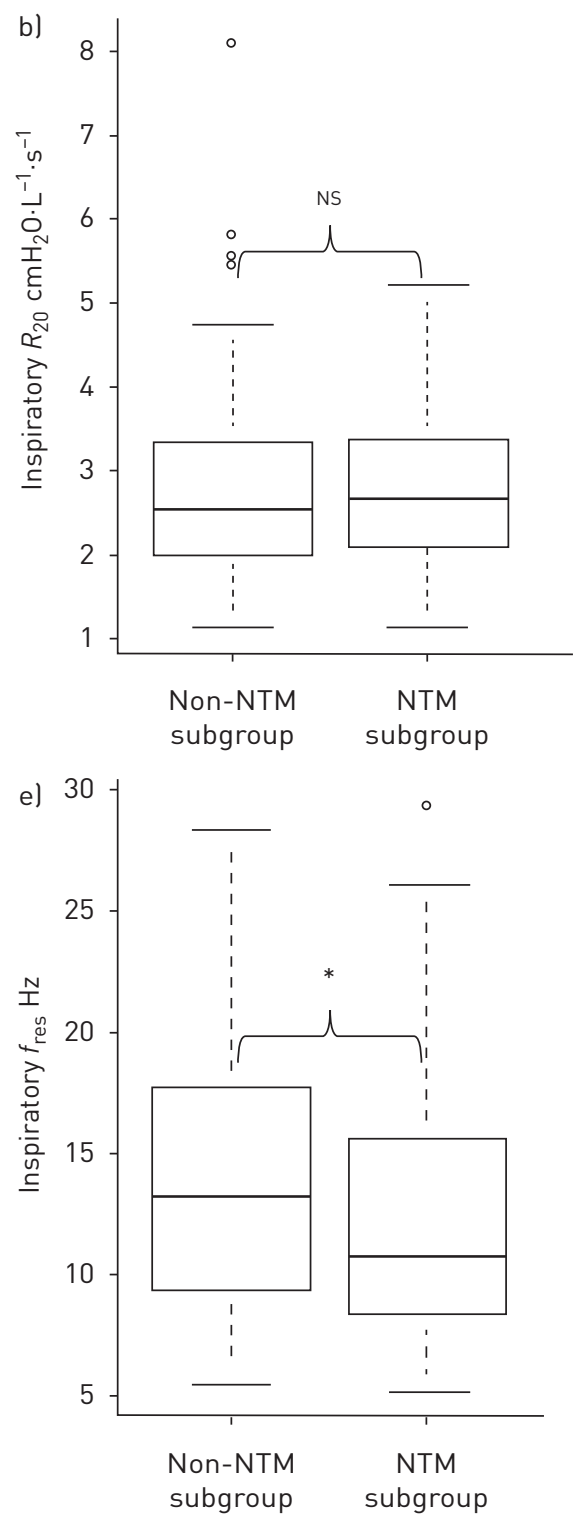
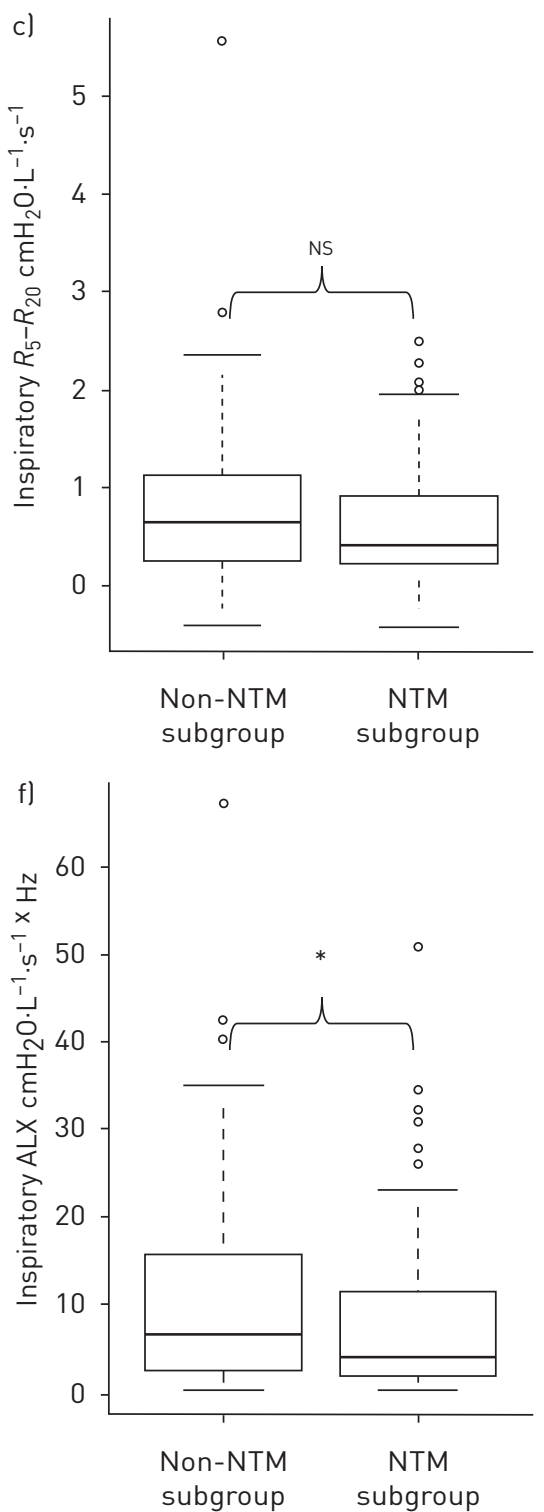

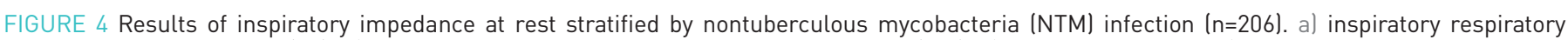
system resistance at $5 \mathrm{~Hz}\left(R_{5}\right)$, b) inspiratory respiratory system resistance at $\left.20 \mathrm{~Hz}\left(R_{20}\right), \mathrm{c}\right)$ inspiratory $R_{5}-R_{20}$, d) inspiratory respiratory system reactance at $5 \mathrm{~Hz}\left(X_{5}\right)$, e) inspiratory resonant frequency $\left(f_{\text {res }}\right)$, and $f$ ) inspiratory low-frequency reactance area (ALX). $N T M$ subgroup ( $\left.=98\right)$ and non-NTM subgroup $(\mathrm{n}=108)$. *: $\mathrm{p}<0.05$ as measured by the Mann-Whitney $U$-test.

TABLE 4 Receiver operating characteristic curve of inspiratory resonant frequency in patients with or without nontuberculous mycobacteria (NTM) infection for predicting hospital admissions and mortality (n=206)

\begin{tabular}{|c|c|c|c|c|c|c|c|}
\hline \multirow[t]{2}{*}{ Subgroup } & \multirow[t]{2}{*}{ Area under curve } & \multirow[t]{2}{*}{ p-value } & \multicolumn{2}{|c|}{$95 \% \mathrm{Cl}$} & \multirow[t]{2}{*}{ Cut-off value } & \multirow[t]{2}{*}{ Sensitivity } & \multirow[t]{2}{*}{ Specificity } \\
\hline & & & Lower limit & Upper limit & & & \\
\hline \multicolumn{8}{|c|}{ Hospital admission within 4 years } \\
\hline Non-NTM subgroup & 0.675 & 0.0008 & 0.570 & 0.779 & 14.180 & 0.709 & 0.642 \\
\hline \multicolumn{8}{|l|}{ Mortality within 4 years } \\
\hline NTM subgroup & 0.819 & 0.0182 & 0.699 & 0.939 & 11.750 & 0.620 & 1.000 \\
\hline
\end{tabular}


admissions and 11.750 for mortality, and 14.180 for hospital admissions and 14.430 for mortality in the non-NTM subgroup). Given the baseline characteristics of the NTM subgroup that were significantly different from the non-NTM subgroup, sex and Pseudomonas colonisation were hypothesised to affect inspiratory $f_{\text {res }}$ (table 3 ). However, only NTM infection, age and pulmonary lobes with bronchiectasis correlated independently with inspiratory $f_{\text {res }}$ (supplementary table 10). Hence, the results showed that the cut-off values of inspiratory $f_{\text {res }}$ for hospital admissions and mortality could be independently affected by NTM infection.

\section{Discussion}

The present study highlights three major findings regarding the usefulness of IOS in bronchiectasis: 1) respiratory impedance measured by IOS, particularly respiratory reactance, is useful for evaluating disease severity; 2) inspiratory but not expiratory impedance is useful for evaluating disease severity; and 3) IOS is useful for evaluating disease severity of bronchiectasis both with and without NTM infection. To the best of our knowledge, this was the largest study that investigates the usefulness of IOS for predicting disease severity in bronchiectasis and the first study that demonstrates the usefulness of IOS in patients with NTM infection.

In bronchiectasis patients, respiratory impedance has been reported to be effective for diagnosis and not to change between convalescence and acute exacerbations [16]. Thus, the present study included bronchiectasis patients regardless of their clinical condition. In addition, it has been demonstrated that respiratory resistance might be an indicator of poorer clinical conditions [16]. In particular, $R_{5}-R_{20}$, which is considered to reflect small airway conditions, was reported to be useful for the diagnosis of bronchiectasis [17]. However, no study has yet reported on the usefulness of each IOS parameter for predicting the disease severity and prognosis in patients with bronchiectasis. Given the results of the present study, both respiratory reactance and $R_{5}-R_{20}$ were demonstrated to be useful for estimating the disease severity, with both statistical and clinical significance. Of note, all the reactance parameters were more useful than the resistance parameters (including $R_{5}-R_{20}$ ) for predicting hospital admissions, mortality and the disease severity indices. Given the correlation of respiratory impedance with spirometric parameters, the usefulness of respiratory reactance might have been attributed to the combined correlations with restrictive ventilation deficiency and airflow obstruction (supplementary table 2). Although, in the present study, IOS and spirometry were not as useful as the BSI for predicting hospital admissions, inspiratory $f_{\text {res }}$ and $\mathrm{FEV}_{1}$ were equally useful as the FACED score as a predictor of hospital admissions and mortality. PFTs, including IOS and spirometry, might be appropriate for monitoring in bronchiectasis because the disease severity indices include variables with small fluctuations (e.g. age and Pseudomonas colonisation). $\mathrm{FEV}_{1}$ declines gradually and thus might be preferred for monitoring in bronchiectasis [25]; however, given the effortless measurement, inspiratory $f_{\text {res }}$ could be useful as a complementary score for evaluating and monitoring disease severity in bronchiectasis patients.

The present study also found that inspiratory impedance correlated better than expiratory impedance with the disease severity of bronchiectasis. Although the mechanism remains unclear, patients with chronic obstructive pulmonary disease, asthma and interstitial lung disease have also been reported to show wider variation in expiratory impedance $[26,27]$. The present study implies that measurement of IOS could be affected by the variation of expiration regardless of patients' aetiology. Therefore, inspiratory impedance might be used when evaluating disease severity in patients with bronchiectasis. However, further investigations are necessary to validate the results and assess the mechanism.

Our results indicate that IOS could be useful for evaluating disease severity in patients with NTM infection, although the cut-off values of inspiratory $f_{\text {res }}$ might be different between the NTM and non-NTM subgroups. MCDonnell et al. [28] reported that patients with right middle-lobe and lingular predominant bronchiectasis have a higher incidence of NTM and a lower BSI and FACED score, suggesting a milder phenotype of bronchiectasis. Moreover, women have additional risk factors for this phenotype, which is attributable to the pulmonary restriction caused by breast tissue or pregnancy [28, 29]. Given the large population with Mycobacterium avium complex (MAC), the relatively focal distribution of bronchiectasis on HRCT might have affected the lower cut-off values of inspiratory $f_{\text {res }}$ in the NTM subgroup. Further studies to validate the hypothesis and improve the clinical usefulness of IOS in bronchiectasis would be desirable.

The present study had some limitations. First, it was a single-centre investigation, and some selection bias might thus have affected the findings. Second, this study mainly included patients with MAC in the NTM subgroup, and the number of patients with other types of NTM infection was small. Hence, a larger study population would be required to validate the results. Finally, the IOS device (i.e. Mostgraph-01), might have affected respiratory impedance, and it is unclear whether the findings of the present study are applicable to other IOS devices. 
In conclusion, this study assessed the correlation of IOS parameters with hospital admissions, mortality and the disease severity indices. Inspiratory $f_{\text {res }}$ measured by IOS could be useful for estimating the disease severity of bronchiectasis both with and without NTM infection, although the cut-off values might be different in patients with NTM infection. Further investigations of bronchiectasis with and without NTM infection are needed.

Acknowledgements: The authors would like to thank S. Ito, S. Sakaguchi and T. Uenishi (Laboratory for Clinical Investigation, National Hospital Organization Osaka Toneyama Medical Center, Toyonaka, Japan) for their help with spirometry and IOS measurements.

Author contributions: Y. Yamamoto contributed to the conceptualisation and design of the study, and data collection and analysis; and wrote the manuscript. K. Miki and K. Tsujino contributed to the design of the study and supervised the manuscript. T. Kuge contributed to the conceptualisation of the study. T. Matsuki, K. Fukushima, Y. Oshitani, H. Kagawa and K. Yoshimura cared for all the patients and contributed to data analyses. M. Miki contributed to design of the study and data interpretation, and supervised the manuscript. H. Kida contributed to supervision of the manuscript. All the authors reviewed and approved the submission of the final manuscript.

Conflict of interest: None declared.

\section{References}

1 Chalmers JD, Hill AT. Mechanisms of immune dysfunction and bacterial persistence in non-cystic fibrosis bronchiectasis. Mol Immunol 2013; 55: 27-34.

2 Cole PJ. Inflammation: a two-edged sword--the model of bronchiectasis. Eur J Respir Dis Suppl 1986; 147: 6-15.

3 McShane PJ, Naureckas ET, Tino G, et al. Non-cystic fibrosis bronchiectasis. Am J Respir Crit Care Med 2013; 188: 647-656.

4 Davies G, Wells AU, Doffman S, et al. The effect of Pseudomonas aeruginosa on pulmonary function in patients with bronchiectasis. Eur Respir J 2006; 28: 974-979.

5 Loebinger MR, Wells AU, Hansell DM, et al. Mortality in bronchiectasis: a long-term study assessing the factors influencing survival. Eur Respir J 2009; 34: 843-849.

6 Chalmers JD, Goeminne P, Aliberti S, et al. The bronchiectasis severity index. An international derivation and validation study. Am J Respir Crit Care Med 2014; 189: 576-585.

7 Chalmers JD, Aliberti S, Filonenko A, et al. Characterization of the "frequent exacerbator phenotype" in bronchiectasis. Am J Respir Crit Care Med 2018; 197: 1410-1420.

8 Martínez-García MÁ, de Gracia J, Vendrell Relat M, et al. Multidimensional approach to non-cystic fibrosis bronchiectasis: the FACED score. Eur Respir J 2014; 43: 1357-1367.

9 Martinez-Garcia MA, Athanazio RA, Girón R, et al. Predicting high risk of exacerbations in bronchiectasis: the E-FACED score. Int J Chron Obstruct Pulmon Dis 2017; 12: 275-284.

10 Rosales-Mayor E, Polverino E, Raguer L, et al. Comparison of two prognostic scores (BSI and FACED) in a Spanish cohort of adult patients with bronchiectasis and improvement of the FACED predictive capacity for exacerbations. PLoS ONE 2017; 12: e0175171.

11 Oostveen E, MacLeod D, Lorino H, et al. The forced oscillation technique in clinical practice: methodology, recommendations and future developments. Eur Respir J 2003; 22: 1026-1041.

12 Tse HN, Tseng CZS, Wong KY, et al. Accuracy of forced oscillation technique to assess lung function in geriatric COPD population. Int J Chron Obstruct Pulmon Dis 2016; 11: 1105-1118.

13 Horsley A, Siddiqui S. Putting lung function and physiology into perspective: cystic fibrosis in adults. Respirology 2015; 20: 33-45.

14 Shirai T, Kurosawa H. Clinical application of the forced oscillation technique. Intern Med 2016; 55: 559-566.

15 Raj D, Sharma GK, Lodha R, et al. Correlation between impulse oscillometry and spirometry parameters in Indian patients with cystic fibrosis. Chron Respir Dis 2014; 11: 139-149.

16 Guan WJ, Gao YH, Xu G, et al. Impulse oscillometry in adults with bronchiectasis. Ann Am Thorac Soc 2015; 12 657-665.

17 Guan WJ, Yuan JJ, Gao YH, et al. Impulse oscillometry and spirometry small-airway parameters in mild to moderate bronchiectasis. Respir Care 2016; 61: 1513-1522.

18 Pasteur MC, Helliwell SM, Houghton SJ, et al. An investigation into causative factors in patients with bronchiectasis. Am J Respir Crit Care Med 2000; 162: 1277-1284.

19 Griffith DE, Aksamit T, Brown-Elliott BA, et al. An official ATS/IDSA statement: diagnosis, treatment, and prevention of nontuberculous mycobacterial diseases. Am J Respir Crit Care Med 2007; 175: 367-416.

20 American Thoracic Society. Standardization of spirometry, 1994 update. American Thoracic Society. Am J Respir Crit Care Med 1995; 152: 1107-1136.

21 Kubota M, Kobayashi H, Quanjer PH, et al. Reference values for spirometry, including vital capacity, in Japanese adults calculated with the LMS method and compared with previous values. Respir Investig 2014; 52: 242-250.

22 Pasteur MC, Bilton D, Hill AT, et al. British Thoracic Society guideline for non-CF bronchiectasis. Thorax 2010; 65: Suppl. 1, i1-58.

23 DeLong ER, DeLong DM, Clarke-Pearson DL. Comparing the areas under two or more correlated receiver operating characteristic curves: a nonparametric approach. Biometrics 1988; 44: 837-845.

24 Kanda Y. Investigation of the freely available easy-to-use software "EZR" for medical statistics. Bone Marrow Transplant 2013; 48: 452-458.

25 Martinez-García MA, Oscullo G, Posadas T, et al. Pseudomonas aeruginosa and lung function decline in patients with bronchiectasis. Clin Microbiol Infect 2020; in press [https://doi.org/10.1016/j.cmi.2020.04.007].

26 Paredi P, Goldman M, Alamen A, et al. Comparison of inspiratory and expiratory resistance and reactance in patients with asthma and chronic obstructive pulmonary disease. Thorax 2010; 65: 263-267. 
27 Sugiyama A, Hattori N, Haruta Y, et al. Characteristics of inspiratory and expiratory reactance in interstitial lung disease. Respir Med 2013; 107: 875-882.

28 McDonnell MJ, Ahmed M, Das J, et al. Patterns of disease in patients with middle-lobe predominant bronchiectasis. Respiration 2017; 93: 406-414

29 Yu JA, Pomerantz M, Bishop A, et al. Lady Windermere revisited: treatment with thoracoscopic lobectomy/ segmentectomy for right middle lobe and lingular bronchiectasis associated with non-tuberculous mycobacterial disease. Eur J Cardiothorac Surg 2011; 40: 671-675. 